\title{
Supporting Information for \\ Contact Conductivity of Uncapped Carbon Nanotubes Formed by Silicon Carbide Decomposition
}

\author{
Masafumi Inaba ${ }^{1}$, Chih-Yu Lee ${ }^{1}$, Kazuma Suzuki ${ }^{1}$, Megumi Shibuya ${ }^{1}$, Miho Myodo ${ }^{1}$, Yu Hirano ${ }^{1}$, \\ Wataru Norimatsu ${ }^{2}$, Michiko Kusunoki ${ }^{2}$, Hiroshi Kawarada ${ }^{1,3}$ \\ 1. Graduate School of Science and Engineering, Waseda University, 3-4-1 Okubo, Shinjuku, Tokyo, \\ 169-8555, Japan \\ 2. EcoTopia Science Institute, Nagoya University, Furo-cho, Chikusa, Nagoya, 464-8603, Japan \\ 3. The Kagami Memorial Laboratory for Materials Science and Technology, Waseda University, \\ 2-8-26 Nishiwaseda, Shinjuku, Tokyo, 169-0051, Japan \\ * M. Inaba Email: inaba-ma@ruri.waseda.jp
}




\section{Derivation of Eq. 3}

To derive Eq. 3, we considered a unit of the conductive mesh in Fig. 5(a), which is shown in Fig. S1 as the area surrounded by the blue rectangle. The lengths of the lateral and vertical sides are $d_{C N T / C N T}$ and $\sqrt{3} d_{C N T / C N T}$, respectively, where $d_{C N T / C N T}$ is the distance between the centers of neighboring CNTs. Assuming that the electrical potentials of the nodes are the same at the same lateral position, for example, $\mathrm{n} 1$ and $\mathrm{n} 4$, and $\mathrm{n} 2$ and $\mathrm{n} 5$, the conductance per unit in the lateral direction in Fig. $\mathrm{S} 1$ is calculated to be $3 g_{C}$. This unit is periodically aligned in the conductive mesh, $L / d_{C N T / C N T}$ units in the lateral direction and $W /\left(\sqrt{3} d_{C N T / C N T}\right)$ units in the vertical direction.

The total conductance $G_{T}$ is written as

$G_{T}=3 g_{C, C N T / C N T} \times \frac{W}{\sqrt{3} d_{C N T / C N T}} / \frac{L}{d_{C N T / C N T}}$.

By simplifying Eq. S1, Eq. 3 is yielded.

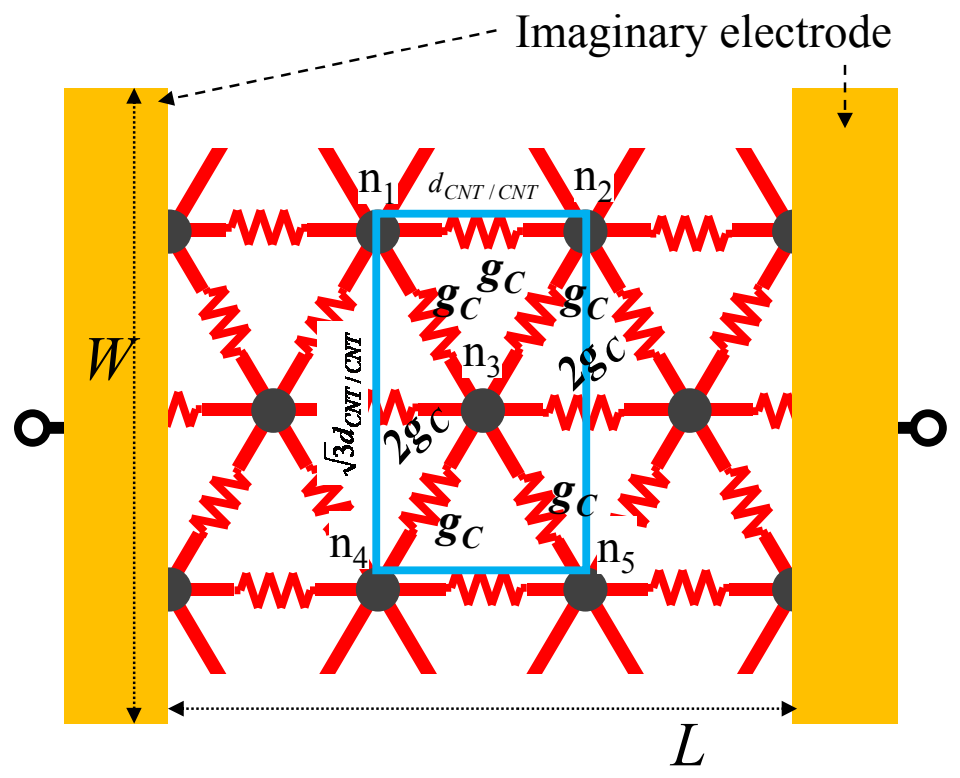

Fig. S1. Approximated conductive mesh of parallel adjacent CNTs with CNT widths and intervals of $W$ and $L$, respectively. The area surrounded by the blue rectangle is a unit of the conductive mesh.

We confirmed that the conductive mesh alignment is not influenced by the imaginary electrode setup. Fig. S2 shows the resistive mesh and imaginary electrode. The difference between Figs. S2 and 5(a) is the arrangement of the electrode. A unit of the conductive mesh is set in the same 
manner as before. The lengths of the lateral and the vertical sides are $\sqrt{3} d_{C N T / C N T}$ and $d_{C N T / C N T}$, respectively. The conductance per unit in the lateral direction in Fig. S2 is calculated to be $g_{C}$. This unit is periodically aligned in the conductive mesh, $L /\left(\sqrt{3} d_{C N T / C N T}\right)$ units in the lateral direction and $W / d_{C N T / C N T}$ units in the vertical direction. The total conductance $G_{T}$ is written as

$G_{T}=g_{C, C N T / C N T} \times \frac{W}{d_{C N T / C N T}} / \frac{L}{\sqrt{3} d_{C N T / C N T}}$.

This equation can also be simplified to Eq. 3 .

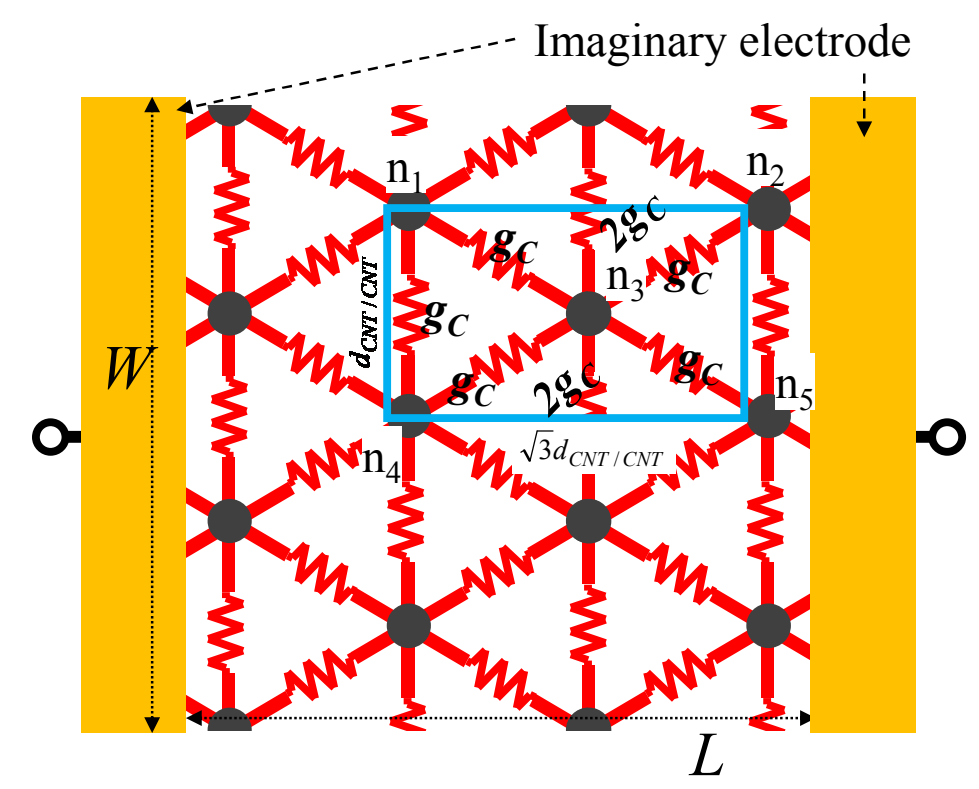

Fig. S2. Approximated conductive mesh of parallel adjacent CNTs in the case that the conductive mesh in Fig. S1 is rotated by $90^{\circ}$. The area surrounded by the blue square is a unit of the conductive mesh. 


\section{Conductivity when CNT forest has vacancies}

To estimate the conductivity of a CNT forest with many vacancies, we assumed the unit of the conductive mesh to be the blue rectangular in Fig. S3. The lengths of the lateral and vertical sides are $2 d_{C N T / C N T}$ and $\sqrt{3} d_{C N T / C N T}$, respectively. The conductance per unit in the lateral direction in Fig. S3 is calculated to be $2 g_{C} / 3$. This unit is periodically aligned in the conductive mesh, $L / 2 d_{C N T / C N T}$ units in the lateral direction and $W /\left(\sqrt{3} d_{C N T / C N T}\right)$ units in the vertical direction. The total conductance $G_{T}$ is written as

$G_{T}=\frac{2}{3} g_{C, C N T / C N T} \times \frac{W}{\sqrt{3} d_{C N T / C N T}} / \frac{L}{2 d_{C N T / C N T}}=\frac{4}{3 \sqrt{3}} g_{C, C N T / C N T} \frac{W}{L}$.

According to this result, the contact conductivity was increased by a factor of $9 / 4$ compared with that for a vacancy free mesh (Fig. S1). However, the structure in Fig. S3 should not be stable because of the CNT vacancies, and the contact width should be enlonged by a factor of 2 . Therefore, the contact conductivity is not significantly affected by the existence of vacancies.

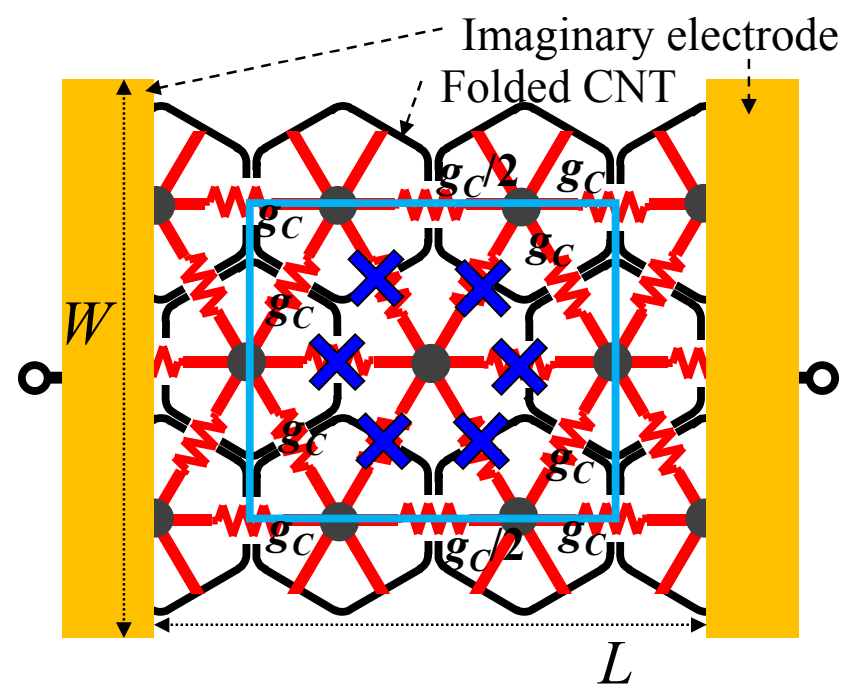

Fig. S3. Conductance mesh of parallel adjacent CNTs with CNT vacancies. The area surrounded by the blue rectangle is a unit of the conductive mesh. 


\section{Contact length of CNT/CNT Y-type contact in dispersed-CNT thin-film transistors}

Znidrasic et al. ${ }^{1}$ reported CNT/CNT contact resistances of approximately $180 \mathrm{k} \Omega$ for $\mathrm{X}$-type connection and $60 \mathrm{k} \Omega$ for Y-type connection. In the case of Y-type contact, parts of the CNTs are in parallel contact with each other. Assuming that the CNT on-axis conduction is $1 \times 10^{-8} \mathrm{~S} / \mathrm{cm}$ and the contact width is $\sim 0.1 \mathrm{~nm}$, the Y-type contact resistance of $60 \mathrm{k} \Omega$ corresponds to a contact length of $\sim 200 \mathrm{~nm}$. We also calculated the contact length for different contact widths. To calculate the contact length of Y-type contact, two patterns were considered. Figs. S4(a) and (b) show the two types of contact, named the Y-type and I-type. For the Y-type contact, the apparent contact resistance $R_{C}^{\prime}$ is

$$
R_{C}^{\prime}=\sqrt{\frac{2 \rho_{C N T} \rho_{C, C N T / C N T}}{w_{C}}} \operatorname{coth}\left(\sqrt{\frac{2 \rho_{C N T} w_{C}}{\rho_{C, C N T / C N T}}} l_{C}\right),
$$

where $\rho_{C N T}$ is the CNT on-axis resistivity, $\rho_{C, C N T / C N T}$ is the CNT/CNT conductivity, $w_{C}$ is the CNT/CNT contact width, and $l_{C}$ is the contact length. Note that the apparent contact resistance $R_{C}^{\prime}$ contains both the CNT/CNT contact resistance and the resistance of CNTs, and is defined as the resistance between the two arrows in Figs. S4(a) and (b). In the same way, for the I-type contact, the apparent contact resistance $R_{C}^{\prime}$ is

$$
R_{C}^{\prime}=\rho_{C N T} l_{C}+\frac{\rho_{C, C N T / C N T}}{w_{C} l_{C}} .
$$

Fig. S4(c) shows the relationship between the apparent contact resistance $R_{C}^{\prime}$ and the contact length $l_{C}$ for various contact widths $w_{C}$. The value of $\rho_{C, C N T / C N T}$ is fixed to $2 \times 10^{-8} \Omega \mathrm{cm}^{2}$, which corresponds to a contact conductivity of $0.5 \times 10^{8} \mathrm{~S} / \mathrm{cm}^{2}$. The solid lines correspond to the Y-type contact and the dashed lines correspond to the I-type contact. When the contact length is less than $\sim 100 \mathrm{~nm}$, the contact resistance of both contacts is almost the same. When the contact length is more than $\sim 500 \mathrm{~nm}$, the contact resistivity is almost unchanged for the Y-type contact but increases for the I-type contact. For thin CNTs, the contact width should be small owing to the CNT stiffness. The contact width of small diameter CNTs is not clear but should be lower than the diameter of the CNTs, i.e., $\sim 1 \mathrm{~nm}$. The actual contact lengths are not mentioned in the literature, but the above range is reasonable. Note that if the contact length is longer than $3 \mu \mathrm{m}$, the contact resistance can be neglected because the CNT on-axis resistivity is dominant. 

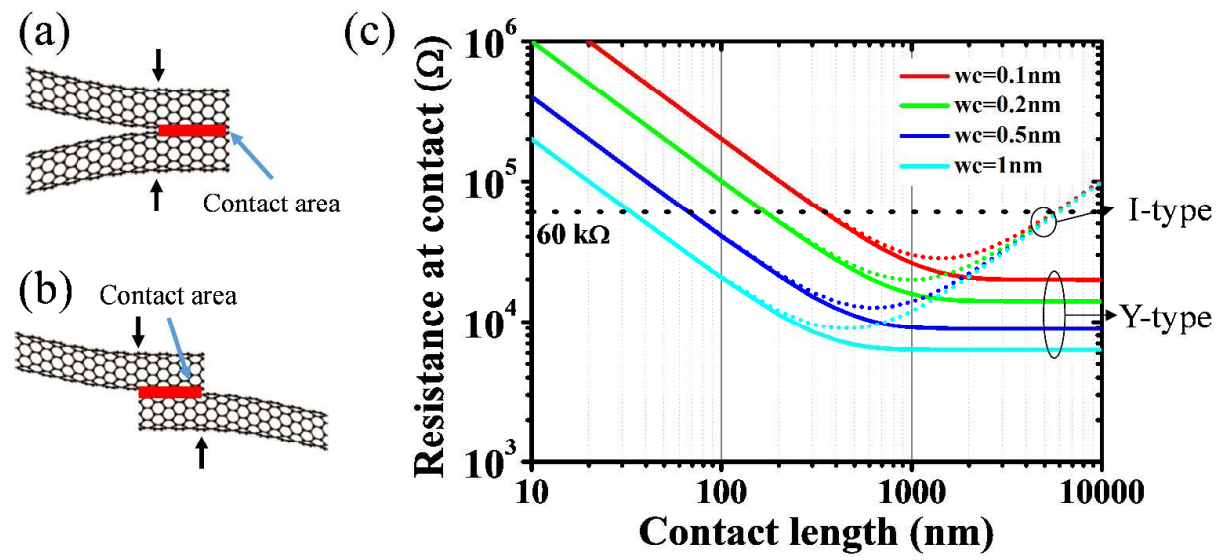

Fig. S4. Schematic image of (a) Y-type contact and (b) I-type contact. The apparent resistance $R_{C}^{\prime}$ corresponds to the resistance between the two arrows. (c) Relationship between contact length and calculated contact resistance of two CNTs for various contact widths for the Y-type and I-type contacts.

\section{REFERENCE}

1 Znidarsic, A.; Kaskela, A.; Laiho, P.; Gaberscek, M.; Ohno, Y.; Nasibulin, A. G.; Kauppinen, E. I.; Hassanien, A. Spatially Resolved Transport Properties of Pristine and Doped Single-Walled Carbon Nanotube Networks. J. Phys. Chem. C 2013, 117, 13324-13330. 